\title{
Journal of Nephrology Research
}

\section{Which is the Role of Leptin on Patients in the End Stage of Renal Disease?}

\author{
Vaia D. Raikou
}

Vaia D. Raikou, $1^{\text {st }}$ Dpartment of Medicine, Propaedaetic, National \& Kapodistrian University of Athens, School of Medicine, Athens, Greece

Correspondence to: Vaia D. Raikou, PhD, $1^{\text {st }}$ Dpartment of Medicine, Propaedaetic, National \& Kapodistrian University of Athens, School of Medicine, Athens, Greece

Email: vraikou@med.uoa.gr

Telephone: +302107456261

Fax: +302107791839

Received: May 15, 2015

Accepted: June 12, 2015

Published online: June 26, 2015

\section{ABSTRACT}

Leptin is a small peptide hormone $(16 \mathrm{kDa})$, a product of the obesity gene $(\mathrm{Ob})$, and is mainly synthesized and secreted by adipocytes.In this review paper the main points of the leptin role on the end stage of renal disease is out lined.

\section{(c) 2015 ACT. All rights reserved.}

Key words: Leptin; Dialysis; Inflammation; Arterial stiffness; Cardiovascular disease

Raikou VD. Which is the Role of Leptin on Patients in the End Stage of Renal Disease? Journal of Nephrology Research 2015; 1(1): 1014 Available from: URL: http://www.ghrnet.org/index.php/jnr/article/ view/1137

\section{INTRODUCTION}

The end-stage of renal disease (ESRD) is characterized by increments of plasma concentrations of hormones produced by adipose tissue known as adipocytokines (including leptin, resistin, tumor necrosis factor-alpha and adiponectin), possibly caused by both passive accumulation from reduced renal excretion and metabolic abnormalities induced by uremia ${ }^{[1]}$. Leptin, which belongs to IL6 family of cytokines, related to diminished food intake. Since leptin is mainly secreted by adipose tissue, serum leptin levels are directly proportional to adipocyte mass. Apart from metabolism, leptin has systemic effects including regulation of angiogenesis, wound healing, lipolysis, blood pressure homeostasis, reproduction, hematopoiesis and immune function ${ }^{[2,3]}$. The current studies provide evidence that the real role of leptin on the patients in end stage of renal disease is complicated rather than clear.

\section{LEPTIN, INFLAMMATION, MALNUTRITION AND INSULIN RESISTANCE}

It has been already recognized that a substantial number of patients with end-stage renal disease have serologic evidence of an activated inflammatory state ${ }^{[4]}$. Previously, the leptin receptor has been identified on endothelial cells, and leptin has been reported to promote both angiogenesis and inflammation ${ }^{[5]}$. Leptin modifies the systemic inflammatory response and the inflammatory microenvironement in renal disease may be associated with elevated expression of leptin gene $^{[6]}$. However, there is a discrepancy for the relationship of leptin levels with chronic inflammation and it has been supported that leptin may be a negative acute phase protein in chronic hemodialysis patients ${ }^{[7]}$. On the other hand, leptin suppresses appetite and increases energy expenditure, playing a homeostatic role in the regulation of food intake and in maintaining body composition in general population ${ }^{[8]}$. In patients with end-stage renal disease malnutrition and hypoalbuminemia are common and powerful predictors of morbidity and mortality in this population ${ }^{[9]}$. One potential relationship between malnutrition and inflammation in renal disease patients is appetite suppression and the link between inflammation and anorexia may be through the leptin ${ }^{[10]}$. However, it has been already supported that inflammation is unlikely to reduce appetite causing malnutrition in dialysis patients through a leptin-mediated mechanism, because leptin is a negative rather than a positive acute phase protein in this population of patients ${ }^{[7]}$. Also, recent study reported that leptin levels were elevated in ESRD patients with normal and mild malnutrition, 
although were lower in severe and moderate category of malnutrition score $^{[11]}$ in agreement with other previous findings ${ }^{[12]}$. The decreased serum levels of leptin in severe category of malnutrition group might be associated with acquired leptin receptor resistance ${ }^{[11]}$.

However, it has been showed that the levels of acyl ghrelin were lowest in the severe group of malnutrition ${ }^{[11]}$. Ghrelin is an appetite stimulating hormone and transduces signals to hypothalamic regulatory nuclei that control energy homeostasis. Ghrelin is shown to enhance food intake in dialysis patients with mild to moderate malnutrition ${ }^{[13]}$. It has been reported that low acyl ghrelin is associated with poor nutritional status and loss of appetite ${ }^{[14]}$. Leptin, ghrelin and uncoupling protein2 (UCP2) gene polymorphisms are the molecules which implicated in the appetite-energy regulatory pathway of the malnutrition-inflammation syndrome in $\mathrm{ESRD}^{[11]}$. UCP2 is an inner mitochondrial protein implicated in thermogenesis and reduction of reactive oxygen species, thus it leades to generation of heat and resting energy expenditure. Elevated functions of UCP2 are associated with increased total fat mass in dialysis patients, which is a protective condition as higher amount of fat signifies more energy stores in these patients ${ }^{[15]}$.

On the other hand, leptin decreases hypothalamic NPY levels and enhances sympathetic activity with hyperinsulinemia, resulting in appetite suppression and modulation of insulin sensitivity ${ }^{[1]}$. High leptin triggers insulin resistance and vice versa ${ }^{[16]}$.

Our studies in dialysis patients showed that the patients with higher leptin serum concentrations had more activated inflammation and insulin resistance, in comparison to the patients with lower leptin values, leptin acting as a positive rather than as a negative acute phase protein ${ }^{[17,18]}$, inversely to the previous reports ${ }^{[7]}$. Also, in our studies the relationship between leptin serum concentrations and inflammation defined by the serum concentrations of high sensitivity C-reactive protein (hsCRP), and insulin resistance defined by homeostasis model assessment insulin resistance (HOMA-IR) was found significantly positive (Figures 1 and 2).

\section{LEPTIN, METABOLIC ACIDOSIS AND ARTERIAL STIFFNESS}

It has been shown using experimental methods, that leptin induces reactive oxygen species (ROS) generation by increasing fatty acid oxidation via a protein kinase A (PKA) activation, which may play an important role in the progression of atherosclerosis in insulinresistant obese diabetic patients ${ }^{[19]}$ and leptin may contribute to oxidative stress and acidosis status of obese patients ${ }^{[20]}$.

Metabolic acidosis is a common condition in end stage renal disease patients resulting in inflammatory stimulation, lipids oxidation and oxidative stress ${ }^{[21]}$. Maintenance dialysis therapies are often not able to completely correct the base deficit. It has already been reported the role of metabolic acidosis on vascular calcification, as the mineral metabolism disturbances act through the existing metabolic acidosis in dialysis patients ${ }^{[22]}$. Even though the influence of acidosis on vascular calcification is complicated, acidosis promotes inflammation of the arterial wall, releasing cytokines that may induce vascular calcification and arterial stiffness ${ }^{[22]}$. Arterial stiffness, assessed as carotid-femoral pulse wave velocity (c-f PWV) and carotid augmentation index (Aix), was associated with abnormalities in the structure or function of the vascular wall and has shown to be an important parameter for the assessment of cardiovascular risk among the markers of arterial disease ${ }^{[23]}$.

Recent study showed that the hyperleptinemia was associated inversely with vasodilatation of resistance arteries in elderly

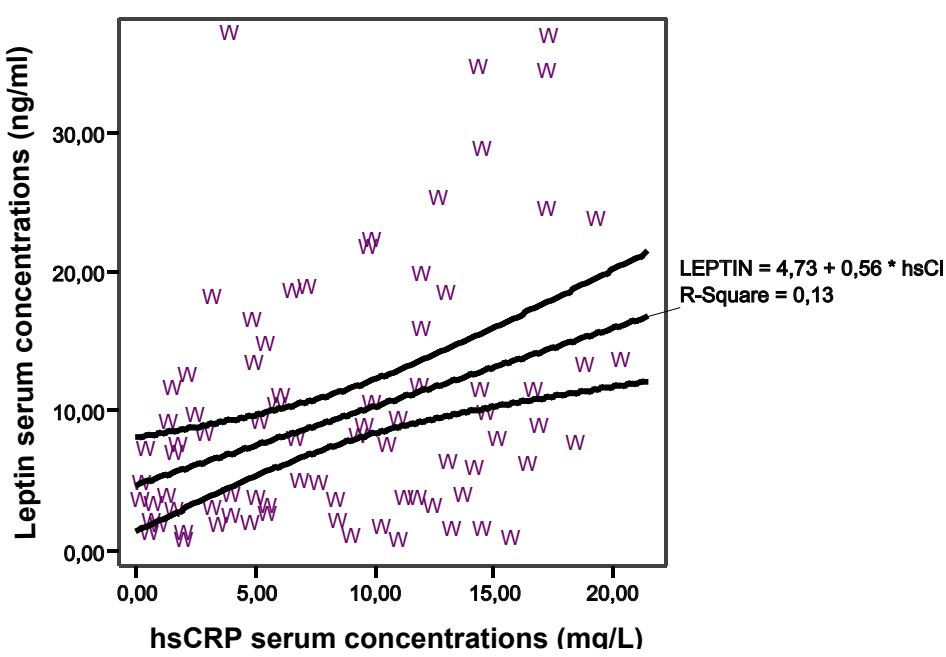

Figure 1 Positive correlation between hsCRP and leptin serum concentrations in dialysis patients $(n=96, p=0.002)$.

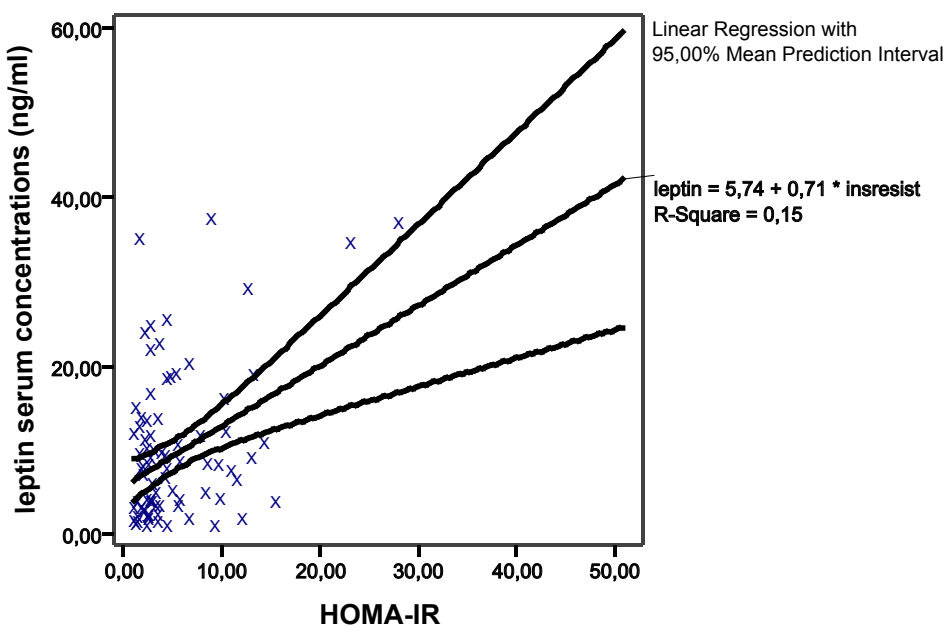

Figure 2 Positive correlation between insulin resistance defined by HOMA-IR and leptin serum concentrations in dialysis patients $(n=96$, $p=0.04)$.

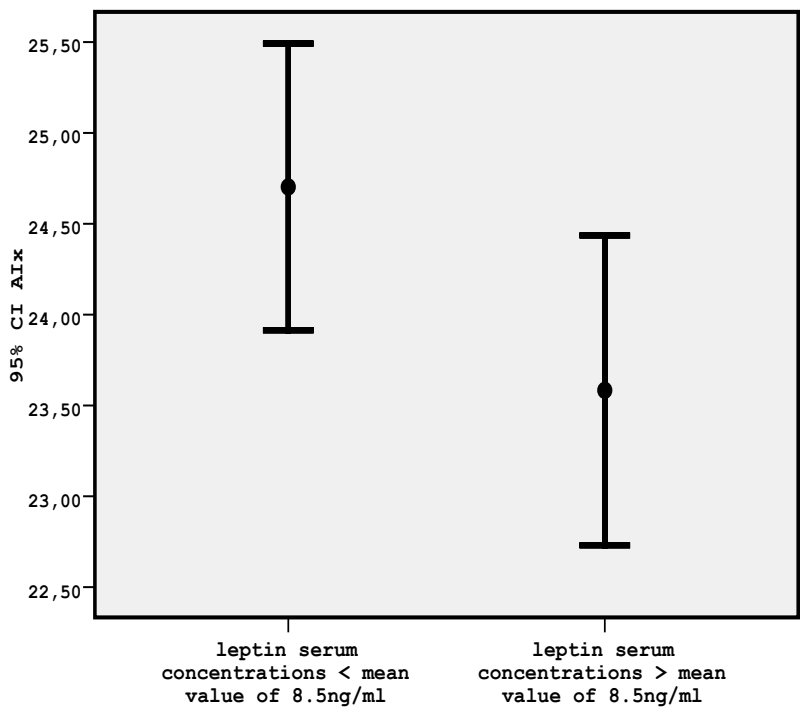

Figure 3 Carotid augmentation index (Aix) values in dialysis patients with less or more than the mean leptin value $=8.5 \mathrm{ng} / \mathrm{mL}(p<0.05)$. 
population of 1,016 subjects $^{[24]}$. Additionally, another previous studies had observed a positive association between c-fPWV, as an indicator of arterial stiffness, and leptin/adiponectin ratio adjusted for gender and age and suggested that leptin regulates the osteoblastic differentiation and calcification of vascular cells and that the artery wall may be an important peripheral tissue target of leptin action $^{[25]}$. Recently, it has been reported that leptin causes endothelial dysfunction and enhances the activity of angiotensin II on blood pressure, activating the sympathetic nervous system and contributing to vascular stiffness and hypertension in obesity ${ }^{[26]}$.

Controversialy to the above reported previous studies, our previous study showed that dialysis patients with higher leptin serum concentrations had a better acidosis state assessed by mildly higher serum bicarbonate levels in combination to lower arterial stiffness, than the patients with lower leptin (Figure 3$)^{[27]}$. However, the previous studies used mainly elderly or obese subjects and not patients on the end stage of renal disease. The group of patients on the end-stage of renal disease, is different than elderly or obese population and in these patients very complicated pathophysiological mechanisms may be activated resulting in a vital balance between multiple inverse reactions. The increased leptin serum concentrations may have an inverse relationship with arterial stiffness, due may to better acidosis status, which in turn may be connected to elevated leptin values in dialysis patients.A such finding may be a reverse causality or a compensatory and protective mechanism versus to the hurtful actions of the increased leptin serum concentrations in these patients. Alternatively, the negative relationship between leptin and arterial stiffness could also show that leptin may be the mediator for the relationship between metabolic acidosis and impaired vascular function in this population of patients.

\section{LEPTIN IN ATHEROSCLEROSIS AND CARDIOVASCULAR DISEASE}

Disorders associated with hyperleptinemia such as obesity and insulin resistance are major risk factors for cardiovascular disease ${ }^{[28]}$. Enhanced chronic systemic inflammation and reduced insulin sensitivity are often associated in patients with chronic renal disease, contributing to cardiovascular morbidity and mortality in these patients $^{[29]}$.

Strong evidence indicates that there is an important role for leptin on hypertension ${ }^{[30]}$. The role of leptin on cardiovascular system to maintain normal blood pressure seem to be balanced in lean individuals with normoglycaemia and leptin sensitivity.Its actions refer to blood pressure lowering mechanisms (via vasodilation, by promoting nitric oxide release from the endothelium), but also to mechanisms that increase blood pressure ${ }^{[31]}$. Controversialy, in obese hyperleptinemic or other leptinresistant conditions as in renal disease, the homeostatic control of blood pressure is impaired. Also, it has been reported the relationship of leprin with atherogenesis, angiogenesis, thrombosis, migration and proliferation of smooth muscle cells ${ }^{[32,33]}$. Previous study using experimental model showed that leptin-deficient mice developed significantly less atherosclerosis and exogenous leptin significantly increased atherosclerosis ${ }^{[34]}$.

Given the above actions, serum leptin levels are reported to be associated with various cardiovascular risks, including stroke, chronic heart failure, acute myocardial infarction ${ }^{[35]}$, coronary heart disease, and left cardiac hypertrophy. Evidence suggests that leptin can stimulate inflammatory response by activating TNF- $\alpha$ via $\mathrm{p} 38$ and JNK MAPK pathway ${ }^{[36]}$ and these inflammatory markers may be associated with the risk of recurrent myocardial infarction and death.
The normal physiological effect of leptin on the regulation of TNF- $\alpha$ expression seems to be suppressive, but the hyperleptinemic condition and leptin resistance may both contribute to the rise of TNF- $\alpha$ in the adipose tissue in obesity ${ }^{[36]}$. Also, several pieces of evidence indicate that TNF- $\alpha$ plays an important role on insulin resistance observed during obesity, which in turn contributes to several pathological problems of obese patients such as hyperlipidemia, arteriosclerosis and hypertension. Because of leptin and TNF- $\alpha$ are overproduced in the adipose tissue of obese individuals and released into the circulation, both may activate inflammatory response and could be involved in the pathogenesis of the CVD during obesity ${ }^{[37]}$.

Additionally, leptin induced expression of monocyte chemoattractant protein-1 (MCP-1), a CC chemokine, which plays an important role in the early phase of atherosclerosis by initiating monocyte/macrophage recruitment to the vessel wall ${ }^{[38]}$, and its expression is actually known to be up-regulated in human atherosclerotic plaques ${ }^{[39]}$.

On the other hand, it has been reported an inverse link between leptin, bone mass and PTH in dialysis patients suggesting that leptin may be implicated in low bone turnover in these patients, likely by the function of central nervous system.Also, leptin may be unrelated to valvular calcification in uremic patients, but it predicts incident cardiovascular events in overweight and obese dialysis patients and it seems to be a connector between bone and cardiovascular alterations in patients with end stage of renal disease ${ }^{[40]}$.

By contrast, some data indicated that leptin may protect against atherosclerosis in specific animal models and human subjects ${ }^{[38]}$. There are reports which revealed that low serum levels of leptin are independent predictors of mortality ${ }^{[12,41]}$. Recent study reported that low serum leptin concentration is an independent risk factor of allcause mortality in stable maintenance hemodialysis patients, but may not be linked with CVD-related mortality ${ }^{[42]}$.

Moreover, another previous study did not support the presence of a clinically relevant relationship between adipocytokines (leptin, adiponectin or resistin) and previous episodes of vascular disease in end stage of renal disease patients, with the only exception of a relationship between resistin levels and heart disease ${ }^{[43]}$.

\section{CONCLUSIONS}

The current data in renal disease patients and also in general population suggests that the net effects of leptin on the pathways of cardiovascular diseases are complicated rather than clear and not completely understood.Leptin may have both, protective and harmful effects on the cardiovascular system. However, the most important risk factor should be the leptin resistance rather than the leptin levels themselves, acting via the stimulation of TNF- $\alpha$ and insulin resistance, which are the main pathophysiological pathways for cardiovascular disease, in combination to some role of bone mass. Despite the elevated leptin levels activate inflammatory process, they are inversely associated with metabolic acidosis status, arterial stiffness and vascular function showing a protective action of leptin in this population of patients.Also, leptin levels are increased mainly in mild than in high malnutrition score and ghrelin may be major marker contributing to malnutrition among ESRD patients, rather than leptin.Further studies on appetite and energy homeostasis regulating markers are required for establishing their role in malnutrition.

\section{CONFLICT OF INTERESTS}

The author declare no conflict of interest. 


\section{REFERENCES}

1. Barazzoni R, Biolo G, Zanetti M, Bernardi A, Guarnieri G.Inflammation and Adipose Tissue in Uremia. J of Renal Nutrition 2006;16(3):204-207.

2. Frühbeck G. Intracellular signalling pathways activated by leptin. Biochem J. 2006;393:7-20.

3. Dubey L, Hesong Z. Role of leptin in atherogenesis. Exp Clin Cardiol.2006;11:269-75.

4. V Panichi, A Scatena, M Migliori,et al. Biomarkers of Chronic Inflammatory State in Uremia and Cardiovascular Disease Int J Inflam. 2012; 2012: 360147.

5. Bouloumie A, Marumo T, Lafontan M, Busse R. Leptin induces oxidative stress in human endothelial cells. FASEB J. 1999;13(10):1231-8.

6. Zoccali C, Tripepi G, Cambareri F, Catalano F,et al. Adipose tissue cytokines, insulin sensitivity, inflammation, and cardiovascular outcomes in end-stage renal disease patients. J Ren Nutr. 2005;15(1):125-30.

7. Burl R Don, Laura M Rosales, Nathan W Levine, William Mitch and George A Kaysen. Leptin is a negative acute phase protein in chronic hemodialysis patients Kidney Int, 2001; 59: 1114-1120.

8. 8.S. Kaur, N. P. Singh, A. K. Jain, and A. Thakur .Serum Creactive protein and leptin for assessment of nutritional status in patients on maintenance hemodialysis. Indian J Nephrol. 2012; 22(6): 419-423.

9. Jahromi SR, Hosseini S, Razeghi E, et al. Malnutrition predicting factors in hemodialysis patients. Saudi J Kidney Dis Transpl. 2010 Sep;21(5):846-51.

10. Bergstrom J: Mechanisms of uremic suppression of appetite. J Ren Nutr 1999;9:129-132.

11. R. Sharma, S. Agrawal, A. Saxena, et al.Association of genetic variants of ghrelin, leptin and UCP2 with malnutrition inflammation syndrome and survival in end-stage renal disease patients. Genes Nutr. 2013; 8(6): 611-621.

12. Scholze A, Rattensperger D, Zidek W, Tepel M. Low serum leptin predicts mortality in patients with chronic kidney disease stage 5 . Obesity, 2007; 15:1617-1622.

13. Wynne K, Giannitsopoulou K, Small CJ, et al. Subcutaneous ghrelin enhances acute food intake in malnourished patients who receive maintenance peritoneal dialysis: a randomized, placebocontrolled trial. J Am Soc Nephrol, 2005; 16:2111-2118.

14. Oner-Iyidogan Y, Gurdol F, Kocak H, et al. Appetite-regulating hormones in chronic kidney disease patients. J Ren Nutr, 2011;21:316-321.

15. Wang X, Axelsson J, Nordfors L, et al.Changes in fat mass after initiation of maintenance dialysis is influenced by the uncoupling protein 2 exon 8 insertion/deletion polymorphism. Nephrol Dial Transplant , 2007;22:196-202.

16. Tsai JP, Tsai CC, Liu HM, Lee CJ, Liou HH, Hsu BG..Hyperleptinaemia positively correlated with metabolic syndrome in hemodialysis patients. Eur J Intern Med. 2011;22(6):e105-9.

17. Vaia D. Raikou, Despina Kyriaki, John N. Boletis .Arterial stiffness and inflammation in patients on hemodialysis. J Mol Pathophysiol 2012; 1(1): 21-28.

18. Kyriaki D, Kanellopoulos PN, Raikou VD. High-density lipoproteins and inflammation in patients on renal replacement therapies. American Journal of Epidemiology and Infectious Disease, 2014; 2 (1): 33-40.

19. Yamagishi SI, Edelstein D, Du XL, Kaneda Y, Guzman M, Brownlee M. Leptin induces mitochondrial superoxide production and monocyte chemoattractant protein-1 expression in aortic endothelial cells by increasing fatty acid oxidation via protein kinase A. J Biol Chem. 2001;276:25096 -25100.

20. 20 .Dong F, Zhang X, Ren J. Leptin regulates cardiomyocyte contractile function through endothelin-1 receptor-NADPH oxidase pathway. Hypertension. 2006; 47(2): 222-229.

21. Kalantar-Zadeh K, Mehrotra R, Fouque D, Kopple JD. Metabolic acidosis and malnutrition-inflammation complex syndrome in chronic renal failure.Semin Dial 2004;17(6):455-65.

22. Al-Aly Z. Vascular calcification in uremia: what is new and where are we going? Adv Chronic Kidney Dis. 2008;15(4):413-9.

23. Pizzi OL, Brandão AA, Pozzan R, Magalhães ME, Campana EM, Fonseca FL, Freitas EV, Brandão AP. Pulse wave velocity, blood pressure and adipocytokines in young adults: the Rio de Janeiro study Arq Bras Cardiol. 2013; 100(1): 60-6.

24. Gonzalez M(1), Lind L, Söderberg S. Leptin and endothelial function in the elderly: the Prospective Investigation of the Vasculature in Uppsala Seniors (PIVUS) study. Atherosclerosis. 2013;228(2): 485-90.

25. Gauthier A, Dubois S, Bertrais S, Gallois Y, Aube C, Gagnadoux $\mathrm{F}$, et al. The leptin to adiponectin ratio is a marker of the number of metabolic syndrome criteria in French adults. J Metabolic Syn. 2012;1:101.

26. Wang J, Wang H, Luo W, Guo C, Wang J, Chen YE, Chang L, Eitzman DT. Leptin-induced endothelial dysfunction is mediated by sympathetic nervous system activity.J Am Heart Assoc. 2013;16;2(5):e000299.

27. VD. Raikou, A. Evagellatou, D. Kyriaki. The relationship between leptin and arterial stiffness in patients on dialysis. J Mol Pathophysiol . 2014; 3(1): 6-10.

28. Li Y, Zhang L, Gu Y, Hao C, Zhu T.Insulin resistance as a predictor of cardiovascular disease in patients on peritoneal dialysis. Perit Dial Int. 2013; 33(4): 411-8.

29. Ian $\mathrm{H}$ de Boer and Rajnish Mehrotra. Insulin resistance in chronic kidney disease: a step closer to effective evaluation and treatment. Kidney International 2014; 86:243-245.

30. Schutte AE and Schutte R. Leptin: a cardiovascular perspective. JEMDSA 2012;17(2):72-76.

31. Beltowski J(1), Wójcicka G, Marciniak A, Jamroz A. Oxidative stress, nitric oxide production, and renal sodium handling in leptin-induced hypertension. Life Sci. 2004;74(24):2987-3000.

32. Koh KK, Park SM, Quon MJ. Leptin and cardiovascular disease: response to therapeutic interventions. Circulation. 2008;117(25):3238-3249.

33. Li L, Mamputu JC, Wiernsperger N, Renier G. Signaling pathways involved in human vascular smooth muscle cell proliferation and matrix metalloproteinase- 2 expression induced by leptin: inhibitory effect of metformin. Diabetes. 2005;54(7):2227-2234.

34. Chiba T, Shinozaki S, Nakazawa T, Kawakami A, Ai M, Kaneko E, Kitagawa M, Kondo K, Chait A, Shimokado K. Leptin deficiency suppresses progression of atherosclerosis in apoE-deficient mice. Atherosclerosis. 2008;196:68 -75.

35. Krasnodebski P, Bak MI, Opolski G, Karnafel W. Leptin in acute myocardial infarction and period of convalescence in patients with type 2 diabetes mellitus. Kardiol Pol. 2010;68:648-53

36. Shen J, Sakaida I, Uchida K, Terai S, Okita K. Leptin enhances TNF- $\alpha$ production via $\mathrm{p} 38$ and JNK MAPK in LPS-stimulated Kupffer cells. Life Sci. 2005;77:1502-15.

37. 37.D Nalini, R Karthick, V Shirin, et al. Role of the adipocyte hormone leptin in cardiovascular diseases - a study from Chennai based Population. Thrombosis Journal 2015, 13:12.

38. Piemonti L, Calori G, Mercalli A, et al. Fasting plasma leptin, tumor necrosis factor-alpha receptor 2, and monocyte chemoattracting protein 1 concentration in a population of glucose-tolerant and glucoseintolerant women: impact on cardiovascular mortality. Diabetes Care 2003;26(10):2883-2889.

39. Nelken NA, Coughlin SR, Gordon D, Wilcox JN. Monocyte chemoattractant protein-1 in human atheromatous plaques. J Clin Invest. 1991;88(4):1121-7.

40. 40.F. Mallamaci, G. Tripepi, C. Zoccali. Leptin in end stage renal disease (ESRD): A link between fat mass, bone and the cardiovascular system. J Nephrol, 2005; 18: 464-468. 


\section{Raikou VD. Leptin and dialysis patients}

41. Ku IA, Farzaneh-Far R, Vittinghoff E, et al. Association of low leptin with cardiovascular events and mortality in patients with stable coronary artery disease: the Heart and Soul Study. Atherosclerosis. 2011;217(2):503-8.

42. X. Bian, Na Liu, Yu Bai, et al. Association of Leptin With Mortality in Patients on Maintenance Hemodialysis. Iranian Journal of Kidney Diseases , 2014; 8.4: 314-20

43. Díez JJ, Iglesias P, Fernández-Reyes MJ, et al. Serum concentra- tions of leptin, adiponectin and resistin, and their relationship with cardiovascular disease in patients with end-stage renal disease. Clin Endocrinol (Oxf). 2005;62(2):242-9.

Peer reviewer: Ming-Jiang $\mathrm{Xu}, \mathrm{MD}, \mathrm{PhD}$, Associate Professor, Laboratory of Liver Diseases, National Institute on Alcohol Abuse and Alcoholism, National Institutes of Health, 5625 Fishers LN, 2S12, Rockville, MD 20852, USA. 